\title{
Pivotal role of hydrogen sulphide in reversal of impaired platelet nitric oxide signaling in coronary artery spasm
}

\author{
Mdhasan Imam ${ }^{1}$, Thanh Nguyen ${ }^{1}$, Irene Stafford ${ }^{1}$, Saifei Liu ${ }^{1}$, Tamila Heresztyn ${ }^{1}$, Yuliy \\ Chirkov $^{1}$, and John Horowitz ${ }^{1}$ \\ ${ }^{1}$ The University of Adelaide
}

April 28, 2020

\begin{abstract}
Background and purpose: The pathophysiology of coronary artery spasm ( CAS), with its associated ischaemic crises, is currently poorly understood, and treatment is frequently ineffective. In view of increasing evidence that platelet- platelet based defects may occur in CAS patients,. we investigated platelet reactivity in CAS patients and whether symptomatic crises reflect activation of platelet-endothelial interactions. Experimental approach: CAS patients were evaluated during acute and/or chronic symptomatic phases, and compared with healthy control subjects. Inhibition of platelet aggregation with ADP by the nitric oxide (NO) donor sodium nitroprusside (SNP), . and plasma levels of syndecan-1 (glycocalyx shedding marker), tryptase (mast cell activation marker), and platelet microparticles were measured. Key Results: Inhibition of aggregation by SNP was impaired in chronic CAS, and tended to deteriorate further during symptomatic crises, while plasma levels of syndecan-1, tryptase and platelet microparticles increased. Infusion of high dose N-acetylcysteine (NAC) plus glyceryl trinitrate rapidly restored platelet responsiveness to SNP and decreased plasma syndecan-1 levels. The effect of NAC on platelet responsiveness to SNP was mimicked in vitro by the H2S donor NaHS. Conversely, inhibition of enzymatic release of H2S attenuated NAC effect. Conclusion and Implications: CAS is associated with substantial impairment of platelet NO signaling. During acute symptomatic exacerbations, platelet resistance to NO is aggravated, together with mast cell activation and damage to both vasculature and platelets. NAC reverses platelet resistance to NO via release of H2S, and reverses glycocalyx shedding during symptomatic crises: this suggests that H2S donors may correct the pathophysiological anomalies underlying CAS.
\end{abstract}
Abbreviations:
$\mathrm{ACh}=$ acetylcholine
$\mathrm{ADP}=$ adenosine diphosphate
$\mathrm{AOAA}=$ aminooxacetic acid
$\mathrm{CAS}=$ coronary artery spasm
$\mathrm{CSFP}=$ coronary slow flow phenomenon
GTN = glyceryl trinitrate
$\mathrm{H}_{2} \mathrm{~S}=$ hydrogen sulphide
$\mathrm{MDA}=$ malondialdehyde
$\mathrm{NAC}=\mathrm{N}$-acetylcysteine
$\mathrm{NO}=$ nitric oxide
$\mathrm{PA}=$ Prinzmetal's Angina 
$\mathrm{PMP}=$ platelet microparticle $(\mathrm{s})$

SD-1 = syndecan-1

$\mathrm{SGC}=$ soluble guanylate cyclase

$\mathrm{SNP}=$ sodium nitroprusside

TIMI $=$ thrombolysis in myocardial infarction

\section{Introduction}

Coronary artery spasm (CAS, variant angina) comprises both Prinzmetal's angina (PA: predominantly large vessel vasospasm) (Prinzmetal et al. , 1959) and the coronary slow flow phenomenon (CSFP: predominantly small coronary artery spasm) (Beltrame et al. , 2003). Both of these disorders are characterized by cyclic fluctuation of symptoms, irrespective of treatment status (Waters et al. , 1983; Sakata et al. , 1996; Kopetz et al. , 2012) and a poor symptomatic response to treatment with sublingual nitrates (Ninomiya et al. , 2008; Morikawa et al. , 2010). CAS occurs frequently, but represents an ongoing clinical challenge both diagnostically and therapeutically (Teragawa et al. , 2018). These difficulties are compounded by incomplete understanding of the pathogenesis of CAS and of its typically cyclical symptomatic course. Previous investigations have generally focused on potential anomalies in vascular reactivity. Although it has always been assumed that the pathogenesis of CAS relates primarily to increased constrictor stimulation and/or responsiveness of coronary vascular smooth muscle, prophylactic treatment with L-channel calcium antagonists (to diminish responses to vasoconstrictor stimuli) has yielded only limited symptomatic benefit (Picard et al. , 2019).

Recently, there have been a number of reports suggesting that pathogenesis of CAS may extend beyond vascular reactivity and be associated with coronary plaque erosion (Shin et al. , 2015) with associated platelet aggregation and frank thrombosis at sites of such fissures and elsewhere within the coronary circulation in CAS patients. Furthermore, a number of studies provided evidence of increased platelet activation and release of platelet-derived vasoactive substances such as $\beta$-thromboglobulin (Ogasawara et al. , 1986), 5hydroxytryptamine (Murakami et al. , 1998), and thromboxane $\mathrm{A}_{2}$ (Lewyet al. , 1979; Robertson et al. , 1980; Hamm et al. , 1987) detected increased concentrations of platelet aggregates in the coronary venous circulation during symptomatic exacerbations of CAS, without evidence of this phenomenon within the systemic circulation. However, (Miyamoto et al. , 2000) ${ }^{(}$were able to detect release of platelet aggregates into the systemic venous circulation following induction of coronary spasm in the cardiac catheterization laboratory with acetylcholine $(\mathrm{ACh})$ provocation. Interestingly, in a wider context, it has recently been shown that activation of platelets can induce interleukin $1 \beta$ - dependent activation of endothelial cells, indicating apotentially primary role for platelets in the pathogenesis of cardiovascular disease crises (Nheket al. , 2017).

Furthermore, associations have been established between CAS and Kounis syndrome (Kounis et al. , 1991), which involves episodic mast cell and platelet activation. Correspondingly, (Forman et al. , 1985) demonstrated that a coronary artery of a CAS patient who had died following an attack was focally infiltrated with adventitial mast cells.

There is also evidence that CAS per se is associated with more extensive inflammatory activation, although the precise mechanisms whereby inflammation induces spasm remain uncertain (Picard et al. , 2019). Systemic inflammatory markers, including C-reactive protein and white blood cell count, were shown by (Ong et al. , 2015b) to be elevated during acute presentation of CAS.

CAS may be engendered in part by impairment of local or systemic generation of, and/or responsiveness to, nitric oxide (NO), which is also the active metabolite of glyceryl trinitrate (GTN). Indeed, the "gold standard" for definitive diagnosis of CAS requires diagnostic coronary injection of acetylcholine (ACh): the induction of vasospasm with ACh implies a net vasoconstrictor effect, despite the NO-releasing effect of ACh. In a murine model of coronary artery spasm, coronary vasodilator effects of ACh changed (reversibly) to constrictor responses in the presence of thiol oxidation (Yamadaet al. , 2013), suggesting that oxidation of SH 
groups on the soluble guanylate cyclase (sGC) molecule might be critically important as a CAS precipitant. Further, inherited defects of endogenous NO generation from endothelial NO synthase (NOS) have also been implicated as potential causes of CAS (Closs et al. , 2012).

One potential link between inflammatory activation and impaired NO signaling would be inflammatory induction of $\mathrm{NO}$ resistance, or diminished end-organ responsiveness to NO, including its vasodilator, antiaggregatory and anti-inflammatory actions (Chirkov et al. , 2001; Willoughby et al. , 2005; Chirkov et al. , 2007; Willoughby et al. , 2012). We have previously shown that NO resistance is present in platelets from patients with both stable and unstable angina pectoris (Chirkov et al. , 2001), and that NO resistance may reflect increased "scavenging" of NO and/or partial inactivation of its intracellular "receptor" soluble guanylate cyclase (sGC) (Chirkov et al. , 2007). Evaluation of the anti-aggegatory effects of NO represents the most convenient method for assessing responsiveness to NO in humans, and has been utilized extensively by us to explore determinants of the presence, extent and prognostic significance (Willoughby et al. , 2005) of NO resistance, and strategies to either ameliorate or circumvent the problem (Chirkov et al. , 2007; Willoughby et al. , 2012).

We now report the results of an investigation into the mechanisms underlying CAS, in both its chronic phases and during acute exacerbations, focusing on the hypotheses that CAS is engendered, at least in part, by impairment of endogenous anti-aggregatory mechanisms, and that these anomalies are exacerbated during symptomatic crises. The results provide evidence to support this hypothesis, shed light on the possibility that abnormal platelet-endothelial interactions are central to the pathogenesis of CAS, delineate the molecular mechanisms underlying both occurrence and exacerbations of CAS, and provide bases for potential therapeutic amelioration of the condition.

\section{Methods}

\subsection{Patients and control subjects: selection}

The investigation compared patients with known CAS, either due to underlying large vessel spasm (PA; $\mathrm{n}=$ $39)$ or to the CSFP $(n=24)$. In all cases this diagnosis was made at coronary angiography, which showed the absence of haemodynamically significant stenoses in epicardial coronary arteries plus either:-

(a) Inducible coronary artery spasm with intracoronary injection of 25 to $100 \mu \mathrm{g}$ of ACh, with a positive test being defined on the basis of a $>75 \%$ reduction in luminal diameter (either focal or diffuse) together with onset of chest pain (Ong et al. , 2012)

or (b) Presence of CSFP, defined as TIMI-2 flow in at least one major epicardial vessel (Beltrame et al. , 2002; Beltrame et al. , 2003).

All patients $(\mathrm{n}=63)$ were subjected to elective peripheral venous blood sampling during chronic treatment of their angina. However, in 16 cases, sampling was also performed during presentation to hospital with prolonged pain, implying the onset of an acute exacerbation. In such cases, blood samples were taken before initiation of emergency treatment measures, 2-12 hours thereafter, and $2-6$ weeks post resolution of symptoms, in order to compare these 3 phases of the disease process.

Treatment during the acute phase of the disease consisted of the intravenous infusion of GTN at the low rate of $2.5 \mu \mathrm{g} / \mathrm{min}$, utilizing non-adsorptive tubing, together with $\mathrm{N}$-acetylcysteine (NAC, $10 \mathrm{~g}$ per 24 hours), which has been shown to potentiate the vasodilator (Horowitz et al. , 1983) and anti-aggregatory (Loscalzo, 1985) effects of NTG, and which has been shown to suppress cyclical coronary vasoconstriction and associated phasic platelet aggregation in a canine model of coronary artery spasm (Foltset al. , 1991).

Healthy control subjects $(\mathrm{n}=31)$ were recruited by advertisement. Clinical characteristics of patients and control subjects are summarized in the Table.

In patients undergoing coronary angiography, blood sample collection was carried out at least 20 minutes post contrast injection. Blood was taken by venesection into plastic tubes containing1:10 volume of citrate 
anticoagulant (2 parts of $0.1 \mathrm{M}$ citric acid to 3 parts of $0.1 \mathrm{M}$ trisodium citrate, $\mathrm{pH} 5$ ) for platelet aggregation studies, and into EDTA Vacutainer tubes for plasma syndecan-1 and tryptase assays.

All human studies were performed in accordance with the Declaration of Helsinki. The study was approved by the Institutional Human Research and Ethics Committee, and informed consent was obtained from all subjects.

\subsection{Platelet aggregometry}

Aggregation in whole blood was examined utilizing a 4-channel Model 700 impedance aggregometer (ChronoLog, Havertown, Pennsylvania, USA) according to the manufacturer's specifications, and the experimental protocol applied was as described by us previously (Nooney et al. , 2015). In brief, tests were performed at $37^{\circ} \mathrm{C}$ and a stirring speed of $900 \mathrm{rpm}$. Blood samples were diluted two-fold with normal saline (final volume 1 $\mathrm{mL}$ ) and pre-warmed for $5 \mathrm{~min}$ at $37^{\circ} \mathrm{C}$. Aggregation was induced with ADP (final concentration of $2.5 \mu \mathrm{M}$ ). The NO donor sodium nitroprusside (SNP, $10 \mu \mathrm{M}$ ) was added to samples 1 min before ADP. Aggregation was monitored continually for $7 \mathrm{~min}$, and responses were recorded for electrical impedance in ohms. Results obtained from aggregometry assays with respect to responses to SNP were evaluated as a percentage of the extent of maximal aggregation in the presence and absence of SNP, and reported as percent inhibition of aggregation. For in vitro experiments examining effects of hydrogen sulphide $\left(\mathrm{H}_{2} \mathrm{~S}\right)$ on anti-aggregatory responses to SNP , NAC and the $\mathrm{H}_{2} \mathrm{~S}$ donor NaHS were added 15 minutes and 2 minutes, respectively, prior to ADP. Inhibitors of $\mathrm{H}_{2} \mathrm{~S}$ formation [aminooxyacetic acid (AOAA, $0.5 \mathrm{mM}$ ) and D,L-propargylglycine (PAG, $3.3 \mathrm{mM})$ ] were pre-incubated with blood samples for 15 minutes prior to ADP.

\subsection{Syndecan-1 assay:}

Plasma concentrations of the glycocalyx component syndecan-1 (SD-1), a marker of glycocalyx shedding activated by acute inflammation (Mulivor et al. , 2004; Nieuwdorp et al. , 2006), were determined by ELISA (Abcam biotechnology, UK). Assay was performed according to the manufacturer's instructions. Briefly, standards and samples were prepared and were pipetted into a 96- well plate coated with primary antibody for SD-1. The plate was incubated at room temperature for $60 \mathrm{~min}$ on a mechanical shaker followed by a wash step and addition of the enzyme streptavidin-HRP. Finally, a color-forming substrate for that enzyme was added in each well and incubated again for 15min in the dark, then absorbance was assessed using a spectrophotometer (Bio-Rad, USA) at 450nm wavelength.

\subsection{Plasma tryptase assay:}

Total plasma tryptase assay was carried out in plasma samples collected from patients in both acute and chronic phases of CAS. Samples were analyzed by SA Pathology (Adelaide, Australia) utilizing UniCAP tryptase assay (Pharmacia Diagnostics, Uppsala, Sweden), via a fluorescence enzyme immunoassay protocol.

\subsection{Platelet microparticles (PMP):}

Plasma concentrations of PMP (Siljander, 2011) were assayed in both stable CAS and during symptomatic crises, and compared with concentrations in control subjects.

Blood (8ml) was collected into citrate-containing cell preparation tubes (CPT Vacutainer, Becton Dickinson), centrifuged at room temperature at $1700 \mathrm{~g}$ for 30 minutes and the supernatant diluted with an equal volume of phosphate buffered saline (PBS) and re-centrifuged multiple times (10mins @500g, 2mins@10000g and finally 45mins @17000g) to isolate a microparticle pellet, as previously described (Pope et al. , 2016). After re-suspension (1ml PBS containing $1 \%$ fetal calf serum, $2.5 \mathrm{mM}$ calcium), microparticles were incubated with Fc blocking agent for 10 minutes (0.1ml; Miltenyi Biotec). Aliquots $(0.1 \mathrm{ml})$ were then incubated

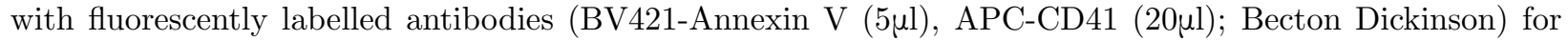
30 minutes at room temperature before addition of counting beads $(25 \mu$ Accucount blank particles [8.0$12.9 \mu \mathrm{m}] ;$ Spherotec) and analyzed via flow cytometry (BD FACS Canto II, Becton Dickinson). Sizing and specificity controls were used to identify populations of microparticles $(1 \mu \mathrm{m})$ positive for Annexin V and platelet microparticles (CD41) staining. 


\subsection{Oxidative stress evaluation:}

Malondialdehyde (MDA) concentrations in plasma were utilized as indices of systemic oxidative stress, and quantified by liquid chromatography-tandem mass spectrometry (LC-MS/MS).

\subsection{Statistical methodology.}

Data were presented as mean values with standard error of mean, or median with interquartile ranges for non-Gaussian data. Unless otherwise stated, one way ANOVA was utilized to assess the differences in distribution among groups with quantitative dependent variables and the Chi-square test for categorical variables. Differences in platelet anti-aggregatory responses to SNP between control subjects and patients were evaluated using ANOVA followed by Bonferroni test, and the measured differences within the patients' blood/plasma samples during acute and chronic or acute and post-NAC phases were assessed by Student's paired t-test. Data comparisons for SD-1 concentrations (acute, with/without NTG/NAC therapy and chronic) and control subjects also utilized ANOVA with post-hoc specific comparisons (Bonferroni test) or Student's paired t-test as appropriate. All tests were 2-tailed and data were expressed as mean \pm SEM, unless otherwise stated; $\mathrm{p}$ values [?] 0.05 were considered statistically significant. Data analyses were performed using SPSS 23 version software.

\section{Results}

\subsection{Patient/control subject characteristics}

In general, this was a middle-aged group of individuals, as summarized in the Table: Patients and control subjects were of similar ages, with a small majority of females. Patients were more likely than control subjects to have histories of hypertension and hyperlipidemia. Patients were typically evaluated during treatment with L-channel calcium antagonists for prophylaxis of angina pectoris. Demographics (data not shown) did not vary significantly according to the type of CAS (PA versus CSFP).

3.2 Platelet reactivity during quiescent ("chronic") phase: CAS patients exhibit hyper-reactivity and impaired responses to SNP

Stable CAS patients exhibited significantly greater aggregability to ADP than control subjects: - mean responses were $8.7+-0.3$ vs $6.5+-0.3 \mathrm{ohms}(\mathrm{p}<0.0001)$. Conversely, there was substantial hyporesponsiveness to SNP. Mean inhibition of ADP-induced platelet aggregation by SNP was $15.7+-1.8$ vs. $32.6+-3.9 \%$ in patients and control subjects respectively ( $<0.0001 ;$ Fig. 1 ).

However, both pro-aggregatory and anti-aggregatory effects did not vary significantly according to the type of CAS diagnosed at coronary angiography. Responses to ADP were $8.9+-0.3$ and $8.3+-0.5$ ohms for PA versus CSFP respectively ( $\mathrm{p}=\mathrm{NS}$ ), and anti-aggregatory effects of SNP, compared in Fig.1, were virtually identical.

\subsection{Acute exacerbation of symptoms is associated with further impairment of NO/sGC sig-} naling.

Evaluation of putative changes in aggregability and responses to SNP between chronic and acute (pre-NAC treatment) phases was made via paired analyses $(\mathrm{n}=14 ;$ Fig. 2$)$. There was a trend $(\mathrm{p}=0.06)$ towards decline in responses to SNP during acute phases of CAS.

\subsection{Plasma syndecan-1 and tryptase concentrations increase during acute symptomatic crises}

We next evaluated potential differences between plasma SD-1 and tryptase concentrations during the acute phase of CAS and those in control subjects, in order to determine whether endothelial glycocalyx shedding was activated, and whether mast cell activation might play a role in this process. During acute exacerbations, SD-1 plasma concentrations were four-fold higher $(\mathrm{p}<0.0001)$ than those in control subjects, and also substantially higher than those seen in the same patients during clinically quiescent periods $(\mathrm{p}<0.0001)$ (Fig. 3A). 
In view of previous evidence linking activation of mast cells with CAS (Forman et al. , 1985; Kounis et al. , 1991), we measured tryptase concentrations in plasma samples from the same patients during chronic and acute phases of CAS. There was a small but consistent increase in plasma tryptase concentrations during acute phases $(\mathrm{n}=10 ; \mathrm{p}=0.03$, Fig. $3 B)$.

However, plasma concentrations of MDA, an index of systemic oxidative stress, did not vary significantly between control subjects $(\mathrm{n}=9 ; 3.05+-0.06 \mu \mathrm{M})$ and patients during either chronic $(3.24 \pm 0.15 \mu \mathrm{M})$ or acute $(3.09 \pm 0.15 \mu \mathrm{M})$ phases of CAS $(\mathrm{n}=9$ for each phase).

\subsection{Symptomatic crises in CAS patients are associated with formation of PMPs.}

PMPs (also known as microvesicles) are particles of less than $1 \mu \mathrm{m}$ diameter which are split from platelet cell membranes under conditions of platelet activation or apoptosis, and interact with vascular endothelium to modulate both inflammatory changes and eventual (Pope et al. , 2016; Rosinska et al. , 2017). In our experiments (Fig. 4), plasma PMP content varied according to group of subjects/patients (ANOVA: $\mathrm{p}=$ $0.03)$, and increased significantly ( $\mathrm{p}=0.02$, Bonferroni post hoc test) among patients evaluated during the acute phase of CAS (Fig. 4 ).

\subsection{GTN/NAC infusion reverses both platelet resistance to NO and SD-1 release during acute phases of CAS.}

Anti-aggregatory effects of SNP were studied in whole blood samples obtained from CAS patients in acute crises, before and during (2-12 hrs) GTN/NAC intravenous infusion (Fig. 5 ). Following the initiation of infusion of GTN/NAC, platelet aggregation in response to ADP decreased marginally from $10.6 \pm 0.5$ to $9.1 \pm 0.5$ ohms $(\mathrm{p}=0.05)$. Furthermore, GTN/ NAC therapy significantly augmented anti-aggregatory responses to $\mathrm{SNP}(\mathrm{n}=11 ; \mathrm{p}=0.003$, Fig. $5 \mathrm{~A})$.

There was a substantial fall in plasma SD-1 concentrations post onset of GTN/NAC treatment $(\mathrm{n}=6 ; \mathrm{p}=$ 0.002 ; Fig. $5 B$ ).

\subsection{Effects of NAC on SNP responses are mediated by formation of $\mathrm{H}_{2} \mathrm{~S}$}

In vitro, in blood samples from patients with chronic CAS, NAC induced concentration-dependent potentiation of the anti-aggregatory effects of SNP. Similar effects were more potently induced by the direct $\mathrm{H}_{2} \mathrm{~S}$ donor NaHS ( $<<0.01$ for both) (Fig. 6 ).

We then assessed the potential impact of inhibitors of $\mathrm{H}_{2} \mathrm{~S}$ formation [PAG (3.3mM) and AOAA (0.5mM)] on NAC-induced potentiation of the anti-aggregatory effects of SNP . There was a significant diminution of SNP effect, both with PAG $(\mathrm{p}=0.0002)$ and with AOAA $(\mathrm{p}=0.001:$ Fig. 7 )

\section{Discussion}

CAS remains a serious clinical problem because, despite recent progress including apparent improvement in the diagnostic process (Elbadawi et al. , 2019), it remains under-diagnosed, its epidemiology is poorly understood, there is no consensus as to its precise pathogenesis, and because the only well-validated prophylactic treatment modality, that of calcium antagonists, yields only moderate symptomatic improvement. The importance of constrictor hyperactivity, in general, to the pathophysiology of CAS, and its link to vascular endothelial dysfunction, has recently been summarized (Ong et al. , 2015a) and is beyond dispute. However, there is also increasing evidence that platelet-based impairment of circulatory homeostasis may be involved (Robertson et al. , 1980; Ogasawara et al. , 1986; Murakami et al. , 1998). In the current study, we investigated whether abnormal platelet reactivity is present in CAS patients and whether symptomatic crises reflect platelet-endothelial interactions. In the current study, we sought to delineate the demographics of patients with CAS, to understand its pathophysiology, and that of the episodic crises which characterize the condition, and to identify a potential "screening test" for acute episodes (to distinguish such episodes from "non-cardiac chest pain"). 
A central basis for our undertaking the current study was continuing uncertainty about the pathogenesis of CAS, despite increasing evidence of vascular hyporesponsiveness to NO (Folts et al., 1991; Yamada et al. , 2013), inflammatory activation (Ong et al. , 2015b) (sometimes involving mast cell activation (Forman et al. , 1985; Kounis et al. , 1991)), a tendency for intracoronary thrombi to be formed at sites of (ill-defined) plaque "erosion" (Shin et al. , 2015), and potentially activation of platelet aggregation (Hamm et al. , 1987; Miyamoto et al. , 2000).

In a series of ex vivo and in vitro experiments, we have now shown that patients with CAS have hyperaggregable platelets which exhibit marked impairment of the anti-aggregatory effects of the NO donor SNP, even during the chronic phases of the disorder. This finding almost certainly has parallels within coronary vascular smooth muscle, which represent the basis for the precipitation of CAS by intracoronary ACh injection (Goto et al. , 1999) . Thus our findings provide a basis for regarding CAS as a combined, biochemicallybased disorder of NO signaling (rather than of NO generation in most cases), differentially affecting both the coronary vasculature and circulating platelets in individual patients (Crea et al. , 2017). Importantly, the current findings appear to apply equally for patients with macrovascular (PA) or microvascular (CSFP) types of CAS.

Platelet aggregatory responses to ADP were greater, and their inhibition by SNP was impaired, in chronic CAS patients relative to control subjects. During symptomatic crises, platelet responses to SNP tended to decrease further, together with substantial release of SD-1 (implying acute damage to the vascular glycocalyx as a contributing factor incremental to endothelial dysfunction). The damaged glycocalyx provides an environment favouring platelet adhesion/activation, with consequent release of pro-constrictor autacoids, such as thromboxane $A_{2}$ and serotonin, which in turn may contribute to coronary vasoconstriction. This is accompanied by elevated plasma tryptase concentrations (implying mast cell activation) and formation of PMPs, the most abundant type of circulating microparticles, the generation of which implies platelet activation and apoptosis (Rosinska et al. , 2017). Overall, these data provide strong evidence that CAS crises are associated with mast cell activation and both vascular and platelet damage, and thus provide a basis for the potential diagnostic utility of SD-1 assay for exclusion of "non-cardiac pain" in patients presenting with acute episodes of CAS.

The finding that intravenous infusion of GTN/NAC reversed the anomalies of platelet SNP responses and of plasma SD-1 concentrations was novel, even though a therapeutic effect based on potentiation of responses to NO was certainly consistent with previous clinical (Pasupathy et al. , 2017) and physiological (Horowitz et al. , 1983; Loscalzo, 1985) studies with GTN/NAC. Although no controlled clinical observations were made of the impact of GTN/NAC infusion on resolution of symptoms, it seems, from the rapid fall in plasma SD-1 concentrations associated with this treatment, that there was associated cessation of acute vascular damage (Mulivor et al. , 2004). These data are reminiscent of the findings of (Foltset al. , 1991) in a canine model of intimal injury to the circumflex coronary artery. These investigators demonstrated that NAC potentiated the effects of GTN in reversing cyclical coronary flow reductions, which resulted from periodic platelet adhesion to the injured vessel wall.

The performance of exploratory in vitro studies shed completely new light on the mechanism of beneficial effect of NAC in a number of acute cardiovascular disease states, with the revelation that potentiation of SNP anti-aggregatory effects by NAC was inhibited in the presence of antagonists (Paul et al. , 2012) of the enzymatic release of $\mathrm{H}_{2} \mathrm{~S}$ from cysteine. Although NAC is known to be a potential donor of $\mathrm{H}_{2} \mathrm{~S}$ (DiNicolantonio et al. , 2017; Bankhele et al. , 2018), and there is also some evidence that $\mathrm{H}_{2} \mathrm{~S}$ and NO may have synergistic effects under some circumstances (Altaany et al. , 2013; Zhou et al. , 2016), the current findings represent the first data to suggest that previously described potentiation of the effects of NO donors by high doses of NAC (Horowitz et al. , 1983; Loscalzo, 1985) may be primarily mediated by $\mathrm{H}_{2} \mathrm{~S}$ release.

In this regard, the current results reinforce those of recent studies suggesting that disordered microvascular reactivity (Levy et al. , 2019) and overt CAS (Ong et al. , 2008) may contribute to the pathogenesis of many forms of acute coronary syndrome. Indeed, the concept that CAS is associated with coronary plaque erosion and associated focal coronary thrombus formation in a substantial minority of cases (Ong et al. , 2015a) 
suggests that at least the acute phases of the disorder are pivotally related to vssel wall: platelet interactions. The current clinically used definition of plaque erosion (Jiaet al. , 2017) includes the presence of thrombus on an apparently intact coronary plaque, but such erosions have been associated with the release into blood of vascular glycocalyx products (Quillard et al. , 2017). These data therefore are consistent with our current findings for acute CAS.

The study has a number of limitations. First, the role of adventitial and/or systemic mast cell activation in precipitation of acute crises remains incompletely delineated. Aggravation of platelet NO resistance during acute crises should be studied in larger cohorts of patients. The precise mechanism(s) whereby NTG/NAC infusion rapidly reverses the glycocalyx shedding which underlies SD-1 release remain uncertain (although there is an implication of decreased generation of at least one "sheddase" enzyme) (Mulivor et al. , 2004), and need both to be correlated with symptomatic effects of this treatment modality and with changes within the cascade of matrix metalloproteinase release associated with mast cell activation. In this regard, it is unfortunate that optical coherence tomography was not performed in CAS patients during acute presentations, in order that plaque erosion and associated focal coronary thrombosis could be diagnosed. The precise mechanisms underlying potentiation of platelet $\mathrm{NO}$ signaling by $\mathrm{H}_{2} \mathrm{~S}$ also remain to be elucidated, although a recent report (Miyamoto et al. , 2017) suggests that combination of $\mathrm{H}_{2} \mathrm{~S}$ and $\mathrm{NO}$ may lead to synergistic vascular effects via polysulphide formation. Finally, the possibility that CAS might fundamentally represent a disorder of $\mathrm{H}_{2} \mathrm{~S}$ generation remains to be explored.

The importance of the current findings therefore rests in three main areas:-

The results reinforce previous, less definitive data, to suggest that fluctuating severity of symptoms in CAS patients reflects, at least in parts, episodic platelet aggregation at sites of vessel wall damage and emphasize that the pathogenesis of CAS is fundamentally related to combined impairment of vasodilator and antiaggregatory mechanisms. These findings, in the acute context, probably represent an indirect reflection of the phenomenon of plaque erosion and associated thrombosis. Clinical findings of plaque erosion (Crea et al. , 2019) and hospital admissions with CAS crises (Elbadawiet al. , 2019) are occurring more frequently. It is also likely that there is a "grey area" of pathogenesis across the whole spectrum of acute coronary syndromes, with plaque erosion increasingly implicated in the pathogenesis of S-T segment elevation acute myocardial infarction (Crea et al. , 2019).

(2) The findings of substantial elevation of SD-1 concentrations during acute attacks could be utilized as a means for provisional diagnosis of CAS in patients with prolonged chest pain who have no definitive changes on ECG or cardiac troponin concentrations. This would reduce the possibility that CAS patients will continue to remain undiagnosed, simply because there is no available screening test.

(3) The finding that there is likely to be an impaired $\mathrm{H}_{2} \mathrm{~S}$ generation and/or a deficient $\mathrm{NO} / \mathrm{H}_{2} S$ interaction during both the chronic and acute phases of the disorder, could be a basis for new therapeutic modalities, both for prophylaxis and treatment of crises. Similarly, the finding of acute mast cell activation carries many potential therapeutic implications (Siebenhaar et al. ,2018). Exploration of such therapeutic options for CAS, coupled with an improvement in diagnostic efficiency, represents a considerable therapeutic priority.

\section{REFERENCES}

Altaany Z, Yang G, Wang R (2013). Crosstalk between hydrogen sulfide and nitric oxide in endothelial cells. J Cell Mol Med 17: 879-888.

Bankhele P, Salvi A, Jamil J, Njie-Mbye F, Ohia S, Opere CA (2018). Comparative Effects of Hydrogen Sulfide-Releasing Compounds on [(3)H]D-Aspartate Release from Bovine Isolated Retinae. Neurochem Res 43: 692-701.

Beltrame JF, Limaye SB, Horowitz JD (2002). The coronary slow flow phenomenon-a new coronary microvascular disorder. Cardiology 97: 197-202.

Beltrame JF, Limaye SB, Wuttke RD, Horowitz JD (2003). Coronary hemodynamic and metabolic studies 
of the coronary slow flow phenomenon. Am Heart J 146: 84-90.

Chirkov YY, Horowitz JD (2007). Impaired tissue responsiveness to organic nitrates and nitric oxide: a new therapeutic frontier? Pharmacol Ther 116: 287-305.

Chirkov YY, Holmes AS, Willoughby SR, Stewart S, Wuttke RD, Sage PR, et al. (2001). Stable angina and acute coronary syndromes are associated with nitric oxide resistance in platelets. J Am Coll Cardiol 37: 1851-1857.

Closs EI, Ostad MA, Simon A, Warnholtz A, Jabs A, Habermeier A, et al. (2012). Impairment of the extrusion transporter for asymmetric dimethyl-L-arginine: a novel mechanism underlying vasospastic angina. Biochem Biophys Res Commun 423: 218-223.

Crea F, Libby P (2017). Acute Coronary Syndromes: The Way Forward From Mechanisms to Precision Treatment.Circulation 136: 1155-1166.

Crea F, Vergallo R (2019). Plaque erosion: Towards precision medicine in acute coronary syndromes. Int $J$ Cardiol 288: 22-24.

DiNicolantonio JJ, JH OK, McCarty MF (2017). Boosting endogenous production of vasoprotective hydrogen sulfide via supplementation with taurine and N-acetylcysteine: a novel way to promote cardiovascular health. Open Heart 4:e000600.

Elbadawi A, Elgendy IY, Naqvi SY, Mohamed AH, Ogunbayo GO, Omer MA, et al. (2019). Temporal Trends and Outcomes of Hospitalizations With Prinzmetal Angina: Perspectives From a National Database. Am $J$ Med.

Folts JD, Stamler J, Loscalzo J (1991). Intravenous nitroglycerin infusion inhibits cyclic blood flow responses caused by periodic platelet thrombus formation in stenosed canine coronary arteries. Circulation 83: 21222127.

Forman MB, Oates JA, Robertson D, Robertson RM, Roberts LJ, 2nd, Virmani R (1985). Increased adventitial mast cells in a patient with coronary spasm. $N$ Engl $J$ Med313: 1138-1141.

Goto A, Ito S, Kondo H, Nomura Y, Yasue N, Suzumura H, et al. (1999). Evaluation of adjunctive intracoronary administration of acetylcholine following intravenous infusion of ergonovine to provoke coronary artery spasm. J Cardiol 34: 309-316.

Hamm CW, Lorenz RL, Bleifeld W, Kupper W, Wober W, Weber PC (1987). Biochemical evidence of platelet activation in patients with persistent unstable angina. J Am Coll Cardiol 10: 998-1006.

Horowitz JD, Antman EM, Lorell BH, Barry WH, Smith TW (1983). Potentiation of the cardiovascular effects of nitroglycerin by N-acetylcysteine. Circulation 68:1247-1253.

Jia H, Dai J, Hou J, Xing L, Ma L, Liu H, et al. (2017). Effective anti-thrombotic therapy without stenting: intravascular optical coherence tomography-based management in plaque erosion (the EROSION study). Eur Heart $J$ 38:792-800.

Kopetz V, Kennedy J, Heresztyn T, Stafford I, Willoughby SR, Beltrame JF (2012). Endothelial function, oxidative stress and inflammatory studies in chronic coronary slow flow phenomenon patients. Cardiology 121: 197-203.

Kounis NG, Zavras GM (1991). Histamine-induced coronary artery spasm: the concept of allergic angina. $\mathrm{Br}$ J Clin Pract 45: 121-128.

Levy BI, Heusch G, Camici PG (2019). The many faces of myocardial ischaemia and angina. Cardiovasc Res115: 1460-1470.

Lewy RI, Wiener L, Smith JB, Walinsky P, Silver MJ, Saia J (1979). Comparison of plasma concentrations of thromboxane B2 in Prinzmetal's variant angina and classical angina pectoris. Clin Cardiol 2: 404-406. 
Loscalzo J (1985). N-Acetylcysteine potentiates inhibition of platelet aggregation by nitroglycerin. $J$ Clin Invest 76: 703-708.

Miyamoto R, Koike S, Takano Y, Shibuya N, Kimura Y, Hanaoka K, et al. (2017). Polysulfides (H2Sn) produced from the interaction of hydrogen sulfide (H2S) and nitric oxide (NO) activate TRPA1 channels. Sci Rep 7:45995.

Miyamoto S, Ogawa H, Soejima H, Takazoe K, Sakamoto T, Yoshimura M, et al. (2000). Formation of platelet aggregates after attacks of coronary spastic angina pectoris.Am J Cardiol 85: 494-497, A410.

Morikawa Y, Mizuno Y, Harada E, Kuboyama O, Yoshimura M, Yasue H (2010). Nitrate tolerance as a possible cause of multidrug-resistant coronary artery spasm. Int Heart J51: 211-213.

Mulivor AW, Lipowsky HH (2004). Inflammation- and ischemia-induced shedding of venular glycocalyx. Am J Physiol Heart Circ Physiol 286: H1672-1680.

Murakami Y, Shimada T, Ishinaga Y, Kinoshita Y, Kin H, Kitamura J, et al. (1998). Transcardiac 5hydroxytryptamine release and impaired coronary endothelial function in patients with vasospastic angina. Clin Exp Pharmacol Physiol25: 999-1003.

Nhek S, Clancy R, Lee KA, Allen NM, Barrett TJ, Marcantoni E, et al. (2017). Activated Platelets Induce Endothelial Cell Activation via an Interleukin-1beta Pathway in Systemic Lupus Erythematosus. Arterioscler Thromb Vasc Biol37: 707-716.

Nieuwdorp M, van Haeften TW, Gouverneur MC, Mooij HL, van Lieshout MH, Levi M, et al. (2006). Loss of endothelial glycocalyx during acute hyperglycemia coincides with endothelial dysfunction and coagulation activation in vivo.Diabetes 55: 480-486.

Ninomiya Y, Hamasaki S, Saihara K, Ishida S, Kataoka T, Ogawa M, et al. (2008). Comparison of effect between nitrates and calcium channel antagonist on vascular function in patients with normal or mildly diseased coronary arteries. Heart Vessels 23: 83-90.

Nooney VB, Hurst NL, Chirkov YY, De Caterina R, Horowitz JD (2015). Post receptor determinants of acute platelet response to clopidogrel in patients with symptomatic myocardial ischemia. Vascul Pharmacol 65-66: 17-22.

Ogasawara K, Aizawa T, Nishimura K, Satoh H, Fujii J, Katoh K (1986). Beta-thromboglobulin release within coronary circulation-a potential role of platelets in ergonovine-induced coronary vasospasm. Int $J$ Cardiol 10:15-22.

Ong P, Athanasiadis A, Hill S, Vogelsberg H, Voehringer M, Sechtem U (2008). Coronary artery spasm as a frequent cause of acute coronary syndrome: The CASPAR (Coronary Artery Spasm in Patients With Acute Coronary Syndrome) Study. J Am Coll Cardiol 52: 523-527.

Ong P, Athanasiadis A, Borgulya G, Mahrholdt H, Kaski JC, Sechtem U (2012). High prevalence of a pathological response to acetylcholine testing in patients with stable angina pectoris and unobstructed coronary arteries. The ACOVA Study (Abnormal COronary VAsomotion in patients with stable angina and unobstructed coronary arteries). J Am Coll Cardiol 59:655-662.

Ong P, Aziz A, Hansen HS, Prescott E, Athanasiadis A, Sechtem U (2015a). Structural and Functional Coronary Artery Abnormalities in Patients With Vasospastic Angina Pectoris. Circ J 79: 1431-1438.

Ong P, Carro A, Athanasiadis A, Borgulya G, Schaufele T, Ratge D, et al. (2015b). Acetylcholine-induced coronary spasm in patients with unobstructed coronary arteries is associated with elevated concentrations of soluble CD40 ligand and high-sensitivity C-reactive protein. Coron Artery Dis 26: 126-132.

Pasupathy S, Tavella R, Grover S, Raman B, Procter NEK, Du YT, et al. (2017). Early Use of N-acetylcysteine With Nitrate Therapy in Patients Undergoing Primary Percutaneous Coronary Intervention for ST-Segment- 
Elevation Myocardial Infarction Reduces Myocardial Infarct Size (the NACIAM Trial [N-acetylcysteine in Acute Myocardial Infarction]). Circulation 136: 894-903.

Paul BD, Snyder SH (2012). H(2)S signalling through protein sulfhydration and beyond. Nat Rev Mol Cell Biol 13: 499-507.

Picard F, Sayah N, Spagnoli V, Adjedj J, Varenne O (2019). Vasospastic angina: A literature review of current evidence. Arch Cardiovasc Dis 112: 44-55.

Pope CA, 3rd, Bhatnagar A, McCracken JP, Abplanalp W, Conklin DJ, O’Toole T (2016). Exposure to Fine Particulate Air Pollution Is Associated With Endothelial Injury and Systemic Inflammation. Circ Res 119: 1204-1214.

Prinzmetal M, Kennamer R, Merliss R, Wada T, Bor N (1959). Angina pectoris. I. A variant form of angina pectoris; preliminary report. Am J Med 27: 375-388.

Quillard T, Franck G, Mawson T, Folco E, Libby P (2017). Mechanisms of erosion of atherosclerotic plaques. Curr Opin Lipidol 28: 434-441.

Robertson RM, Robertson D, Friesinger GC, Timmons S, Hawiger J (1980). Platelet aggregates in peripheral and coronary-sinus blood in patients with spontaneous coronary-artery spasm.Lancet 2: 829-831.

Rosinska J, Lukasik M, Kozubski W (2017). The Impact of Vascular Disease Treatment on Platelet-Derived Microvesicles. Cardiovasc Drugs Ther 31: 627-644.

Sakata K, Yoshida H, Hoshino T, Kurata C (1996). Sympathetic nerve activity in the spasm-induced coronary artery region is associated with disease activity of vasospastic angina. J Am Coll Cardiol 28: 460-464.

Shin ES, Ann SH, Singh GB, Lim KH, Yoon HJ, Hur SH, et al. (2015). OCT-Defined Morphological Characteristics of Coronary Artery Spasm Sites in Vasospastic Angina.JACC Cardiovasc Imaging 8: 1059-1067.

Siebenhaar F, Redegeld FA, Bischoff SC, Gibbs BF, Maurer M (2018). Mast Cells as Drivers of Disease and Therapeutic Targets. Trends Immunol 39: 151-162.

Siljander PR (2011). Platelet-derived microparticles - an updated perspective. Thromb Res 127 Suppl 2: S30-33.

Teragawa H, Oshita C, Ueda T (2018). Coronary spasm: It's common, but it's still unsolved. World J Cardiol 10: 201-209.

Waters DD, Bouchard A, Theroux $\mathrm{P}$ (1983). Spontaneous remission is a frequent outcome of variant angina. $J$ Am Coll Cardiol 2: 195-199.

Willoughby SR, Stewart S, Holmes AS, Chirkov YY, Horowitz JD (2005). Platelet nitric oxide responsiveness: a novel prognostic marker in acute coronary syndromes. Arterioscler Thromb Vasc Biol 25: 2661-2666.

Willoughby SR, Rajendran S, Chan WP, Procter N, Leslie S, Liberts EA, et al. (2012). Ramipril sensitizes platelets to nitric oxide: implications for therapy in high-risk patients. J Am Coll Cardiol 60: 887-894.

Yamada S, Saitoh S, Machii H, Mizukami H, Hoshino Y, Misaka T, et al. (2013). Coronary artery spasm related to thiol oxidation and senescence marker protein-30 in aging. Antioxid Redox Signal 19: 1063-1073.

Zhou Z, Martin E, Sharina I, Esposito I, Szabo C, Bucci M, et al. (2016). Regulation of soluble guanylyl cyclase redox state by hydrogen sulfide. Pharmacol Res111: 556-562.

\section{Figure legends}

Figure 1: Inhibition of ADP-induced platelet aggregation by SNP $(10 \mu \mathrm{M})$ in whole blood samples from healthy control subjects and patients in chronic phase of coronary artery spasm (CAS). Comparisons between coronary slow flow phenomenon (SCFP) and Prinzmetal's angina (PA) subgroups of patients are also shown. 
Figure 2: Comparison of the inhibitory effects of SNP $(10 \mu \mathrm{M})$ on ADP-induced platelet aggregation during acute exacerbations of CAS, compared by paired analysis with the chronic state. There is a trend towards further impairment of SNP responses $(\mathrm{p}=0.06)$ in the acute phase.

Figure 3: (A) Plasma concentrations of SD-1, evaluated in control subjects, and CAS patients in acute and chronic phases. (B ) Ratios of individual plasma tryptase concentrations in acute phase, compared with those in chronic phase $(\mathrm{p}=0.03)$.

Figure 4: Comparison of platelet-derived microparticle (PMP) counts between CAS patients (during acute and chronic phases) and control subjects. ANOVA: $\mathrm{p}=0.03$; Bonferroni test (acute versus chronic phases): $\mathrm{p}=0.02$.

Figure 5: Impact of infusion of NTG/NAC in acutely symptomatic patients:- (A) Impact on ex vivo platelet response to SNP. (B) Impact on plasma SD-1 concentrations

Figure 6: In vitro effects of increasing concentrations of NAC and of the $\mathrm{H}_{2} \mathrm{~S}$ donor NaHS on antiaggregatory responses to SNP $(10 \mu \mathrm{M})$ in blood samples from patients in chronic phase of CAS. ANOVA: $\mathrm{p}$ $<0.01$ for both concentration-response relationships and effect of NaHS versus that of NAC.

Figure 7: Impacts of co-incubation in vitro with inhibitors of $\mathrm{H}_{2} \mathrm{~S}$ formation [PAG (3.3mM) and AOAA $(0.5 \mathrm{mM})]$ on anti-aggregatory responses to SNP $(10 \mu \mathrm{M}) / \mathrm{NAC}(100 \mu \mathrm{M})$ in blood samples from patients in chronic phase of CAS.

Table: Demographics and treatment regimens at time of assessment of coronary artery spasm patients and control subjects recruited ( $\mathrm{p}$ values are for control subjects vs chronic CAS patients).

\begin{tabular}{lllll}
\hline Clinical characteristics & Control subjects & Chronic & Acute & p value \\
\hline Age (years: mean $\pm \mathrm{SD})$ & $58 \pm 9$ & $(\mathrm{n}=63)$ & $(\mathrm{n}=16)$ & \\
Gender (Male; \%) & 42 & $56 \pm 14$ & $56 \pm 12$ & $\mathrm{NS}$ \\
CAD risk factors & & 33 & 40 & $\mathrm{NS}$ \\
Current smoker (\%) & 3 & & & \\
Hypertension (\%) & 19 & 14 & 13 & $\mathrm{NS}$ \\
Diabetes (\%) & 3 & 49 & 40 & 0.007 \\
Hyperlipidemia (\%) & 29 & 15 & 13 & $\mathrm{NS}$ \\
Medications (\%) & & 53 & 40 & 0.05 \\
Aspirin & 7 & & & \\
-Blockers & 0 & 47 & 27 & 0.0001 \\
Ca ${ }^{++}$antagonists & 10 & 14 & 7 & 0.05 \\
ACE-inhibitors/ARB & 23 & 70 & 80 & 0.0001 \\
Statins & 29 & 43 & 33 & 0.07 \\
\hline
\end{tabular}

$\mathrm{ACE}=$ angiotensin converting enzyme; $\mathrm{ARB}=$ angiotensin receptor blockers; $\mathrm{CAD}=$ coronary artery disease; 


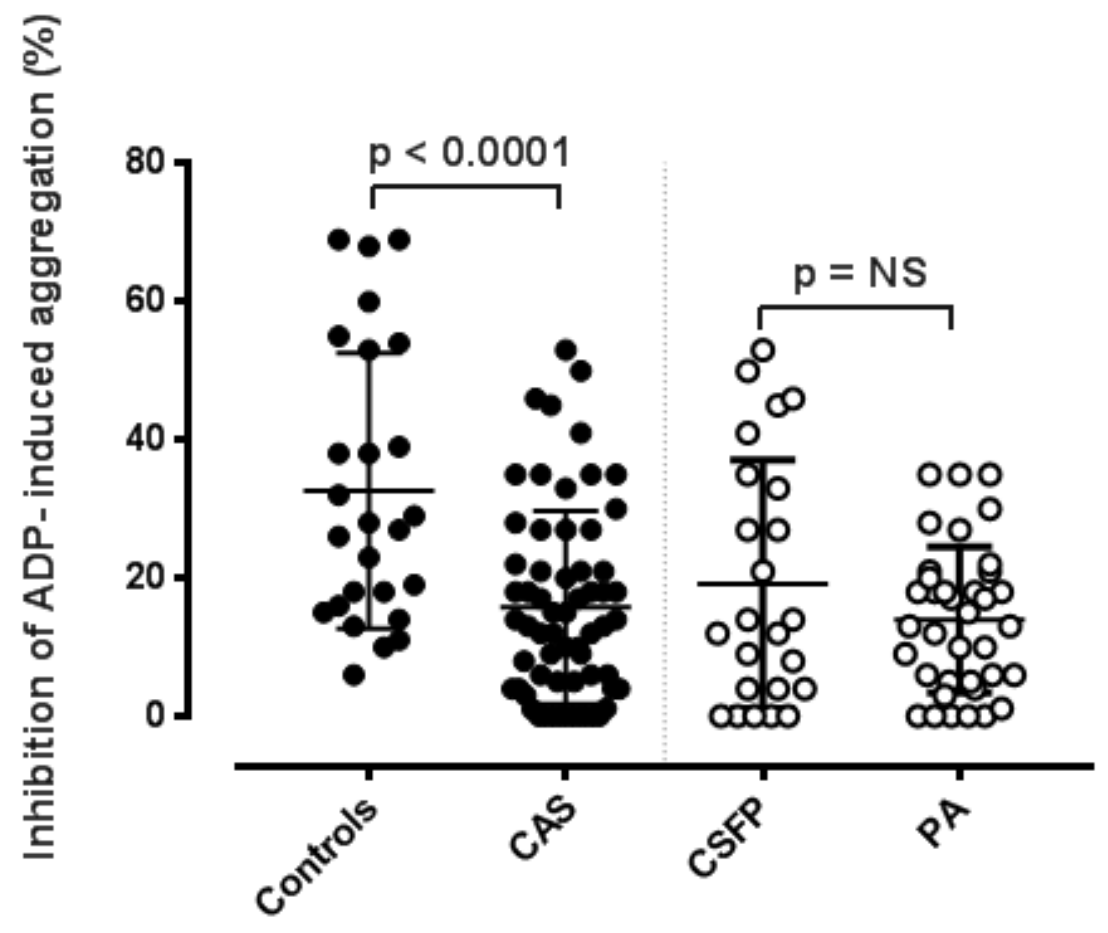

Figure 1: Inhibition of ADP-induced platelet aggregation by SNP $(10 \mu \mathrm{M})$ in whole blood samples from healthy control subjects and patients in chronic phase of coronary artery spasm (CAS). Comparisons between coronary slow flow phenomenon (SCFP) and Prinzmetal's angina (PA) subgroups of patients are also shown. 


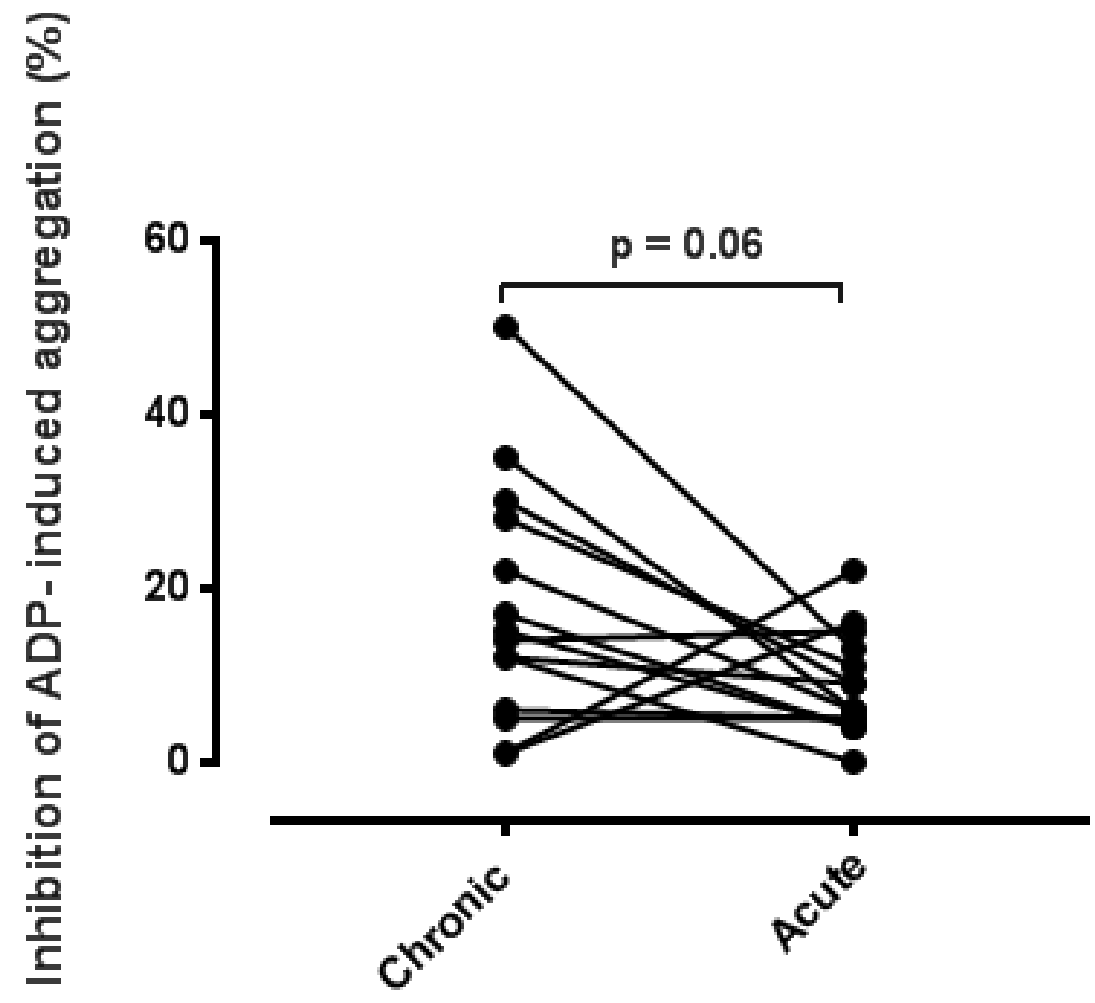

Figure 2: Comparison of the inhibitory effects of SNP $(10 \mu \mathrm{M})$ on ADP-induced platelet aggregation during acute exacerbations of CAS, compared by paired analysis with the chronic state. There is a trend towards further impairment of SNP responses $(p=0.06)$ in the acute phase.
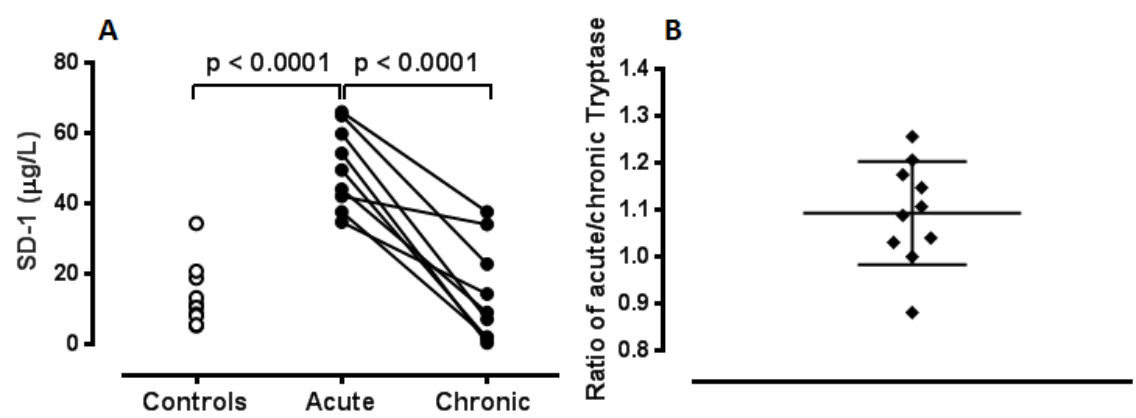

Figure 3: (A) Plasma concentrations of SD-1, evaluated in control subjects, and CAS patients in acute and chronic phases. (B ) Ratios of individual plasma tryptase concentrations in acute phase, compared with those in chronic phase $(\mathrm{p}=0.03)$. 


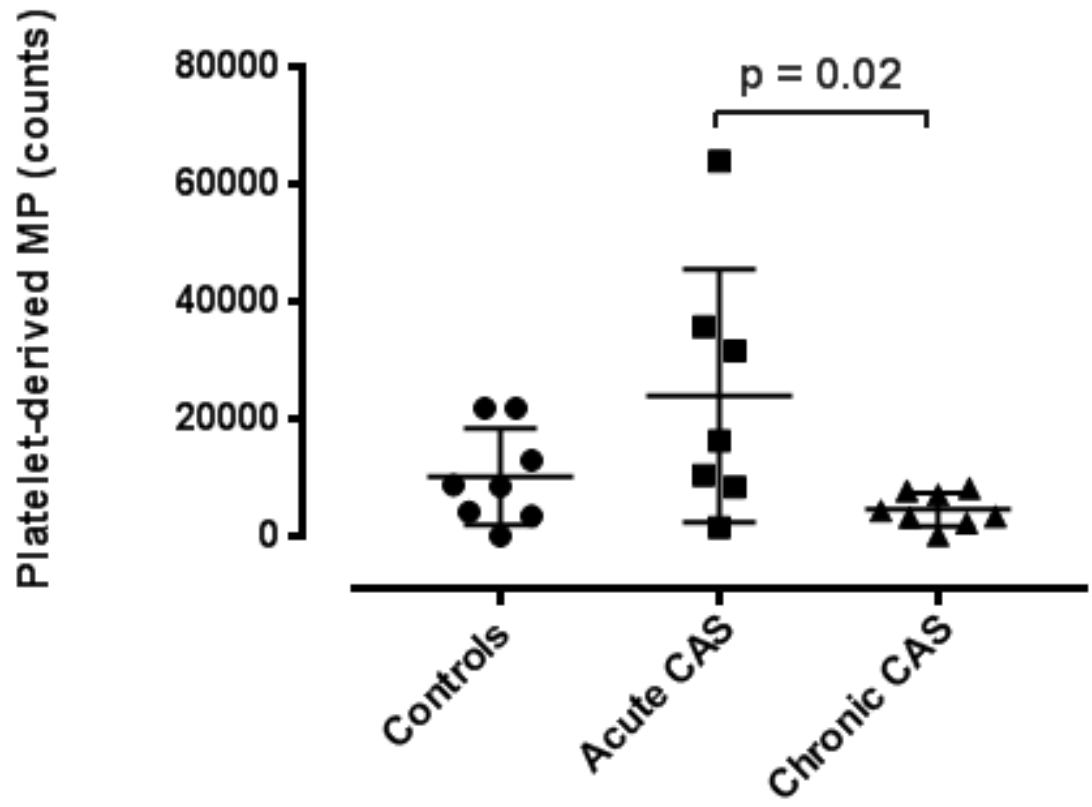

Figure 4: Comparison of platelet-derived microparticle (PMP) counts between CAS patients (during acute and chronic phases) and control subjects. ANOVA: $\mathrm{p}=0.03$; Bonferroni test (acute versus chronic phases): $\mathrm{p}=0.02$. All patients with CAS were receiving treatment with calcium channel blocking drugs, which have been reported to inhibit release of platelet-derived microparticles (Rosinska,Lukasik and Kozubski, 2017).
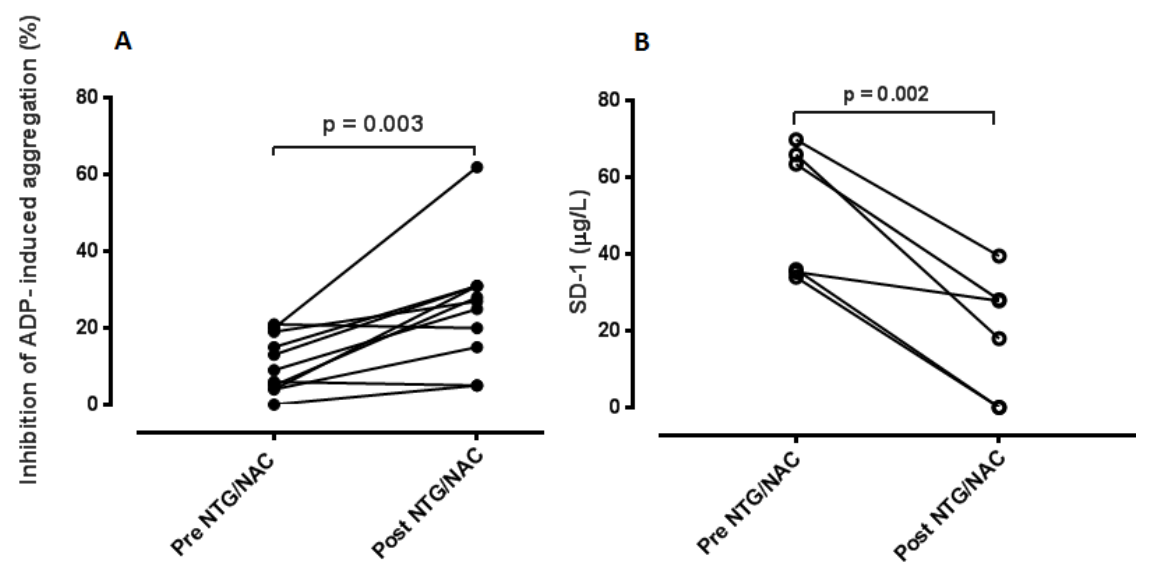

Figure 5: Impact of infusion of NTG/NAC in acutely symptomatic patients:- (A) Impact on ex vivo platelet response to SNP. (B) Impact on plasma SD-1 concentrations 


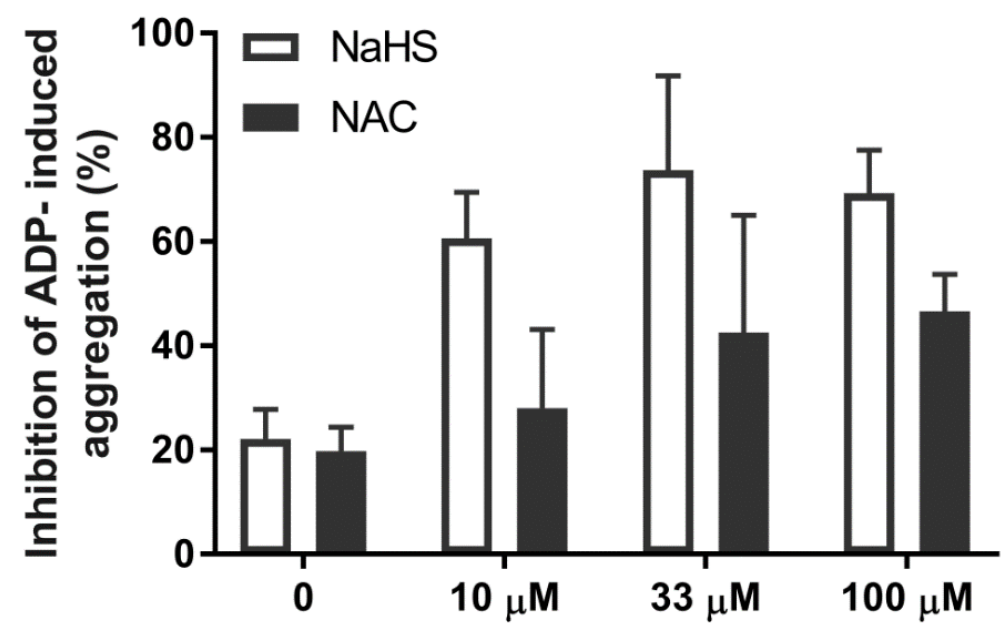

Figure 6: In vitro effects of increasing concentrations of NAC and of the $\mathrm{H}_{2} \mathrm{~S}$ donor NaHS on antiaggregatory responses to SNP $(10 \mu \mathrm{M})$ in blood samples from patients in chronic phase of CAS. ANOVA: p $<0.01$ for both concentration-response relationships and effect of NaHS versus that of NAC.

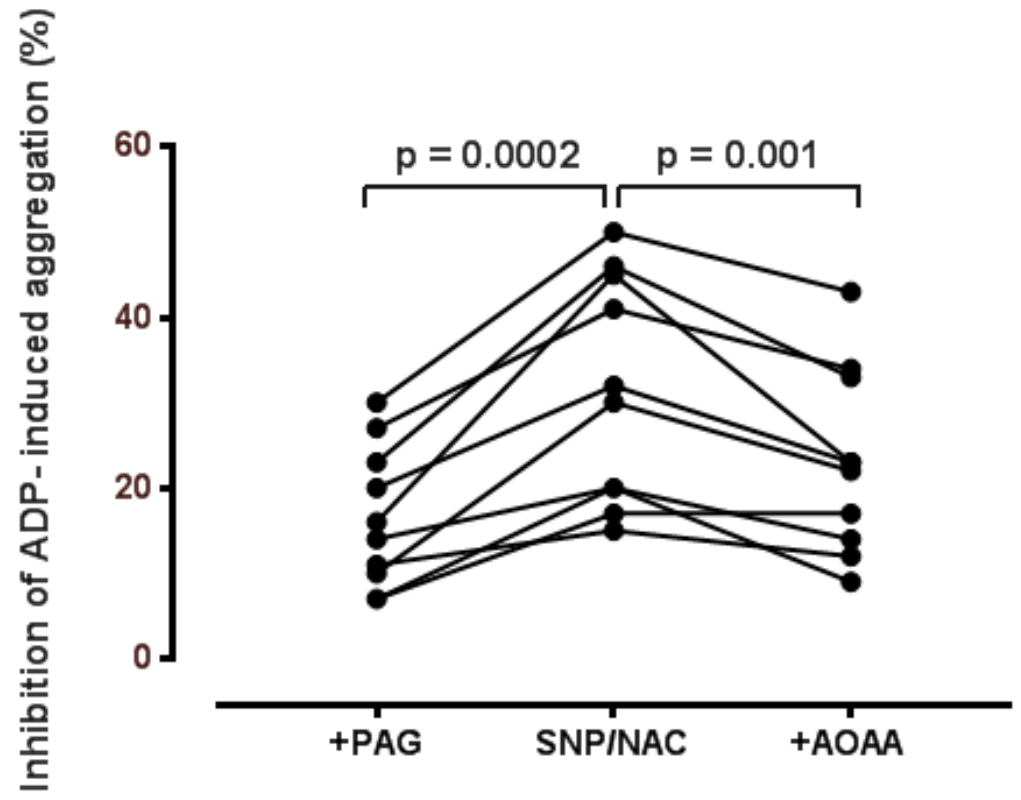

Figure 7: Impacts of co-incubation in vitro with inhibitors of $\mathrm{H}_{2} \mathrm{~S}$ formation $[\mathrm{PAG}(3.3 \mathrm{mM})$ and AOAA $(0.5 \mathrm{mM})]$ on anti-aggregatory responses to $\mathrm{SNP}(10 \mu \mathrm{M}) / \mathrm{NAC}(100 \mu \mathrm{M})$ in blood samples from patients in chronic phase of CAS.

Graphical Abstract 


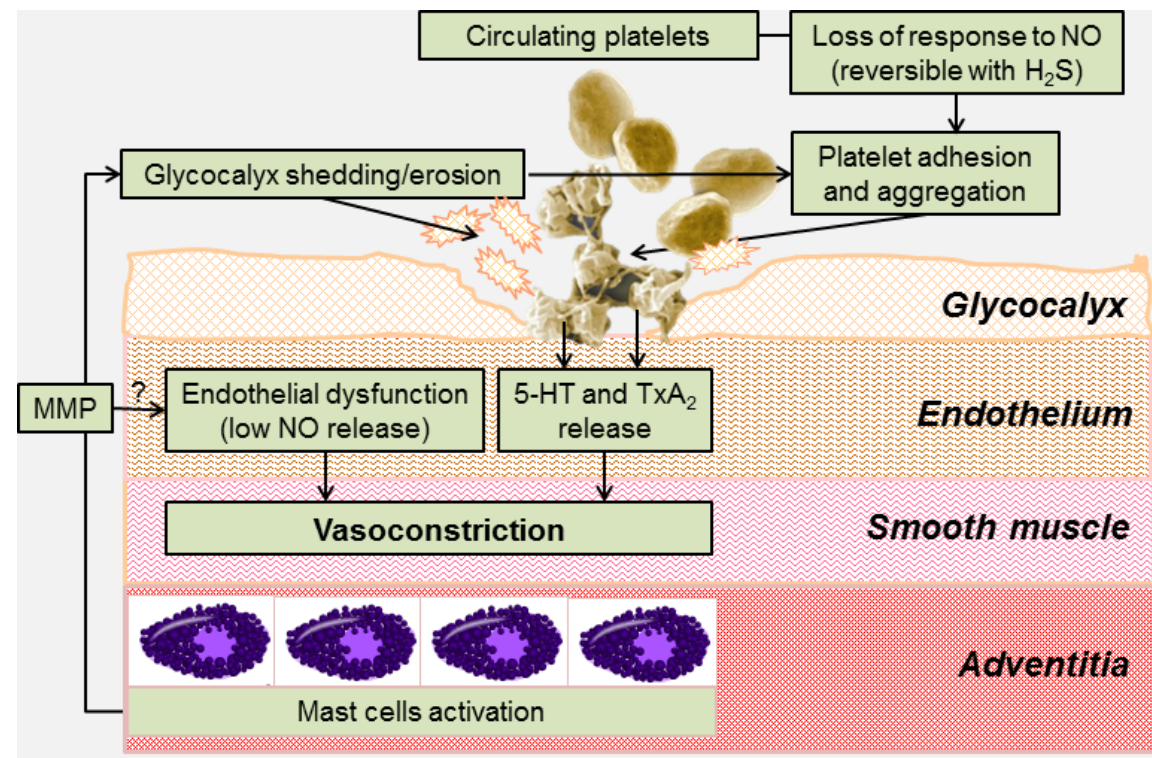

Coronary artery spasm (acute phase): mechanisms

Coronary artery spasm (acute phase): mechanisms.

$\mathrm{MMP}=$ matrix metalloproteinases; $\mathrm{NO}=$ nitric oxide; $\mathrm{TxA}_{2}=$ thromboxane $\mathrm{A}_{2}$;

5 -HT $=5$-hydroxytryptamine (serotonin). 